\title{
Cyclovirobuxine D Attenuates Doxorubicin-Induced Cardiomyopathy by Suppression of Oxidative Damage and Mitochondrial Biogenesis Impairment
}

\author{
Qian Guo, ${ }^{1,2}$ Jiabin Guo, ${ }^{1}$ Rong Yang, ${ }^{1}$ Hui Peng, ${ }^{1}$ Jun Zhao, ${ }^{1} \mathrm{Li} \mathrm{Li},{ }^{2}$ and Shuangqing Peng ${ }^{1}$ \\ ${ }^{1}$ Evaluation and Research Centre for Toxicology, Institute of Disease Control and Prevention, Academy of Military Medical Sciences, \\ 20 Dongdajie Street, Fengtai District, Beijing 100071, China \\ ${ }^{2}$ Department of Biochemistry and Molecular Biology, Chongqing Medical University, Chongqing 400016, China
}

Correspondence should be addressed to Shuangqing Peng; pengsq@hotmail.com

Received 4 November 2014; Revised 11 February 2015; Accepted 23 February 2015

Academic Editor: Ricardo Gredilla

Copyright (C) 2015 Qian Guo et al. This is an open access article distributed under the Creative Commons Attribution License, which permits unrestricted use, distribution, and reproduction in any medium, provided the original work is properly cited.

\begin{abstract}
The clinical application of doxorubicin (DOX) is compromised by its cardiac toxic effect. Cyclovirobuxine D (CVB-D) is a steroid alkaloid extracted from a traditional Chinese medicine, Buxus microphylla. Our results showed that CVB-D pretreatment markedly attenuated DOX-induced cardiac contractile dysfunction and histological alterations. By using TUNEL assay and western blot analysis, we found that CVB-D pretreatment reduced DOX-induced apoptosis of myocardial cells and mitochondrial cytochrome c release to cytosol. CVB-D pretreatment ameliorated DOX-induced cardiac oxidative damage including lipid peroxidation and protein carbonylation and a decrease in the ratio of reduced glutathione (GSH) to oxidized glutathione (GSSG). Moreover, CVB-D was found to prevent DOX-induced mitochondrial biogenesis impairment as evidenced by preservation of peroxisome proliferatoractivated receptor $\gamma$ coactivator-1 $\alpha$ (PGC-1 $\alpha$ ) and nuclear respiratory factor 1 (NRF1), as well as mitochondrial DNA copy number. These findings demonstrate that CVB-D protects against DOX-induced cardiomyopathy, at least in part, by suppression of oxidative damage and mitochondrial biogenesis impairment.
\end{abstract}

\section{Introduction}

Doxorubicin (DOX) is an anthracycline antibiotic which is frequently used to treat a variety of human hematological and solid tumors, such as leukemia and breast cancer $[1,2]$. However, the clinical use of this potent anticancer drug is greatly limited by its concurrent adverse cardiac effect that may ultimately lead to cardiomyopathy and heart failure. It has been reported that more than a quarter of patients receiving DOX develop significant cardiac morbidity [3, 4]. The clinical symptom of DOX-induced cardiomyopathy may manifest as transient arrhythmias, nonspecific electrocardiographic abnormalities, pericarditis, and acute heart failure $[5,6]$. Over the past decades, the development of pharmacological therapy for prevention and/or treatment of the cardiac toxic effect by DOX has become a critical issue in chemotherapy and cardiology. DOX cardiotoxicity involves complex multifactorial processes [5, 7]. Although the exact pathogenesis of DOX-induced cardiotoxicity is not fully understood, oxidative damage and mitochondrial biogenesis impairment have been increasingly proposed as primary mechanisms responsible for DOX-induced cardiotoxicity [2, 5]. DOX induces generation of reactive oxygen species via redox cycling during its intracellular metabolism and leads to wide spread cellular dysfunction and, ultimately, cell death in cardiomyocytes [8]. The onset and severity of DOXinduced cardiac injury have been shown to correlate with the oxidative damage and disruption of mitochondrial biogenesis $[9,10]$. Studies using antioxidant therapy or transgenic mouse overexpressing antioxidant enzymes have shown protection from DOX-induced oxidative damage and cardiotoxicity, which further support a critical role of oxidative stress in DOX cardiotoxicity [11-13]. 
Cyclovirobuxine D (CVB-D; molecular formula: C26H46-N2-O; molecular weight: 402.662; chemical name: 9, 19-cyclopregnan-16-ol, 4, 4, 14-trimethyl-3, 20-bis (methylamino)-, $(3 \beta, 5 \alpha, 16 \alpha, 20 S)-)$ is a triterpenoid alkaloid extracted from a traditional Chinese medicine, Buxus microphylla, which has been widely used for hundreds of years in China to prevent and/or treat many cardiovascular diseases such as arrhythmias, heart failure, and myocardial ischemia [14-17]. As a main active ingredient of Buxus microphylla, increasing clinical and laboratory evidences indicate that CVB-D is an efficient protective agent in the cardiovascular system [14, 17-20]. For instance, double blind clinical trials have reported that CVB-D improves left ventricular function in coronary heart disease $[20,21]$. In laboratory studies, the cardioprotective effect has been shown in animal models including dogs, pigs, and rats $[14,18,19]$. In addition, the beneficial effect of CVB-D has also been shown in vitro cultured ventricular cardiomyocytes and endothelial cells $[14,22,23]$. Although the mechanism of CVB-D's cardioprotective action remains poorly understood, it is increasingly reported that CVB-D may exert antioxidant property $[18,23$, 24]. An in vitro study by $\mathrm{Hu}$ et al. has shown that CVB-D alleviates DOX-induced decrease of cell viability in neonatal rat cardiomyocytes [23], suggesting a protective effect of CVB-D against the cardiac injury by DOX. However, whether CVB-D is protective against DOX-induced cardiotoxicity in vivo is not yet clear. To this end, the present study was designed to test the ability of CVB-D on DOX-induced cardiotoxicity and further explore the mechanism by which CVB-D exerts cardioprotective effect.

\section{Materials and Methods}

2.1. Animals and Drug Treatments. Adult C57BL mice (18 $22 \mathrm{~g}$ ) were purchased from the Animal Center, the Academy of Military Medical Sciences (Beijing, China). Animals were housed in a ventilated animal room maintained at $23 \pm 2.5^{\circ} \mathrm{C}$ with a standard 12/12 light/dark cycle. Food and tap water were provided ad libitum. All experiments were performed according to protocols approved by the Institutional Animal Care and Use Committee in compliance with the Guide for the Care and Use of Laboratory Animals published by the US National Institutes of Health. For each independent experiment, 24 mice were randomly divided into 4 groups, that is, vehicle group, CVB-D group, DOX group, and CVB$\mathrm{D}+\mathrm{DOX}$ group. Three males and three females were included in each group.

Previous studies have shown that CVB-D exerts cardioprotective effects at doses of $0.5-2.0 \mathrm{mg} / \mathrm{kg} / \mathrm{d}$ in rodent models [25]. In clinical studies, the recommended dose of CVB-D for cardiovascular diseases is $1-6 \mathrm{mg} / \mathrm{d}$ [26]. Based on these studies, we pretreated mice daily with CVB-D (purity > 99\%; ZeLang Medical technology Co., Nanjing, China; dissolved in normal saline) by gavage at a dose of $1 \mathrm{mg} / \mathrm{kg} / \mathrm{d}$ for consecutive 4 days (cumulative dose of $4 \mathrm{mg} / \mathrm{kg}$ ) or equal volume of normal saline. On the fifth day, mice were injected intraperitoneally with doxorubicin hydrochloride (HaiSun Pharmaceutical Co., Ltd., Zhejiang, China) at a dose of $15 \mathrm{mg} / \mathrm{kg}$ or equal volume of saline. Four days after DOX treatment, heart function was recorded, and mice were then euthanized by $\mathrm{CO}_{2}$ inhalation. Cardiac tissues were rapidly collected for further examinations.

2.2. Heart Function. Mouse cardiac function was evaluated by a noninvasive echocardiography. Briefly, animals were placed on a warming pad at $37^{\circ} \mathrm{C}$ and anesthetized by $1-$ $2 \%$ isoflurane. Echocardiography measurements were performed by a blinded investigator using M-mode recording at mid-papillary muscle level. Two-dimensional shortaxis images were obtained with a high resolution MicroUltrasound system (Vevo 770, VisualSonics Inc., Canada) equipped with RMV707 mechanical scan probe of $45 \mathrm{MHz}$. The left ventricular end-diastolic diameter (LVEDD) and end-systolic diameter (LVESD), fractional shortening, and ejection fraction were measured and calculated with Vevo Analysis software (version 3.0.0).

2.3. Histological Observation. Hearts subjected to electron microscopic examination were fixed in situ by vascular perfusion with normal saline for $15 \mathrm{~min}$, followed by a $4 \%$ buffered formalin for $10 \mathrm{~min}$. Cardiac tissues were fixed in $4 \%$ buffered formalin for at least $48 \mathrm{~h}$. Afterward, cardiac tissues were embedded in paraffin and sectioned at $4 \mu \mathrm{m}$. The sections were processed using standard histological techniques and further stained with hematoxylin-eosin. Images were captured by a digital imaging system (DP71, OLYMPUS, Japan) connected to a computerized microscopy (BX61, OLYMPUS, Japan).

2.4. Apoptosis Evaluation by TUNEL Assay. Paraffin-embedded sections of heart tissues were prepared and processed for a terminal deoxynucleotidyl transferase-mediated dUTP nick end labeling (TUNEL) assay using an In Situ Cell Apoptosis Detection Kit (Roche, Shanghai, China) according to the manufacturer's instruction. Both negative control and positive control were prepared to display accuracy and objectivity. For each sample, at least 5 fields were randomly selected from 3 slides to quantitatively analyze the apoptotic cells. Data were expressed as the percentage of apoptotic cells by calculating the ratio of positive-staining nuclei to the total number of nuclei.

2.5. Mitochondrion and Cytosol Isolation. Cardiac mitochondrial and cytosolic fractions were isolated from fresh collected heart by differential centrifugation as previously described [27]. In brief, heart tissues were minced and homogenized in an ice-cold extraction buffer $(0.01 \mathrm{M}$ Tris$\mathrm{HCl}, 1 \mathrm{M}$ mannitol, $200 \mathrm{mM}$ sucrose, and $1 \mathrm{mM}$ EGTA; $\mathrm{pH}$ 7.4). The homogenate was centrifuged twice at $600 \mathrm{~g}$ for $5 \mathrm{~min}$ to separate out the nuclear pellet. The supernatant was then centrifuged at $3,000 \mathrm{~g}$ for $10 \mathrm{~min}$ to obtain mitochondrial pellet, and the resulting supernatant was centrifuged at $14,000 \mathrm{~g}$ for $10 \mathrm{~min}$ to obtain cytosolic pellet. These pellets were resuspended and spun again to purify mitochondrial and cytosolic fractions. These isolated fractions were further used for western blot analysis of cytochrome c. The purity of isolated fraction was confirmed by western blot analysis of porin and histone $\mathrm{H} 1$ markers, respectively, for mitochondria 
and nuclei, to show the absence of mitochondrial and nuclear contamination in cytosol fraction and nuclear contamination in mitochondrial fraction.

2.6. Lipid Peroxidation. Cardiac lipid peroxidation was quantified by measuring the formation of thiobarbituric acid reactive substances (TBARS) as we previously described [28]. The TBARS content was measured spectrophotometrically at $532 \mathrm{~nm}$ and calculated based on a standard curve using 1,1,3,3tetraethoxypropane as a standard. Data were expressed as nanomoles per milligram of protein. Protein concentration in the samples was measured by a bicinchoninic acid commercial kit (Biyuntian Co., Hangzhou, China), using bovine serum albumin as a standard.

2.7. Protein Carbonylation. Protein carbonyl content in cardiac tissues was determined according to Floor and Wetzel with slight modification [29]. Briefly, homogenate prepared from fresh heart was reacted with $10 \mathrm{mM} \mathrm{2,4-}$ dinitrophenylhydrazine in $2 \mathrm{M} \mathrm{HCl}$ for $1 \mathrm{~h}$ at room temperature, precipitated with $6 \%$ trichloroacetic acid, and suspended in ethanol/ethyl acetate $(1: 1)$. Proteins were then solubilised in $6 \mathrm{M}$ guanidine hydrochloride and $50 \%$ formic acid was added and centrifuged at $14,000 \mathrm{~g}$ for $10 \mathrm{~min}$ to remove any trace of insoluble material. Carbonyls were measured spectrophotometrically at $370 \mathrm{~nm}$. Data were expressed as nmol of 2,4-dinitrophenyl-hydrazine incorpo$\mathrm{rated} / \mathrm{mg}$ protein based on the molar extinction coefficient of $22,000 \mathrm{M}^{-1} \mathrm{~cm}^{-1}$.

2.8. GSH/GSSH Ratio Determination. Total soluble reduced glutathione (GSH) and oxidized glutathione (GSSG) were measured in fresh heart homogenate using a commercial kit (Jiancheng Bio Ins., Nanjing, China) according to the manufacturer's instruction. Briefly, cardiac tissue homogenates were added to GSH or GSSH assay buffers. For GSH sample, a reaction mixture was added containing $\mathrm{NADPH}, 5,5^{\prime}$ dithio-2-nitrobenzoic acid (DTNB), and glutathione reductase. The rate of DTNB reduction was monitored at the absorbance of $412 \mathrm{~nm}$. For detection of GSSG content, the thio-scavenging reagent 1-methy-2-vinylpyridinium trifluoromethanesulfonate was immediately mixed with the samples to eliminate GSH. GSH and GSSG concentrations were calculated by linear regression against the standard curve and normalized to protein content, and GSH/GSSG ratio was calculated as [(GSH - 2GSSG)/GSSG].

2.9. Western Blot Analysis. Protein samples were extracted from cardiac tissues. Afterward, extracted proteins were electrophoresed and separated on a $12 \%$ SDS-polyacrylamide gel and transferred to a polyvinylidene fluoride membrane (Millipore, Billerica, USA). The membrane was incubated overnight with specific primary antibodies against cytochrome c (Santa Cruz; dilution, 1:2000); PGC-1 $\alpha$ (Abcam, UK; dilution, 1:1000); and nuclear respiratory factor 1 (NRF-1) (Abcam; dilution, 1:1000), TFAM (Abcam; dilution, $1: 1000$ ), MnSOD (Abcam; dilution, 1:1000), UCP2 (Abcam; dilution, $1: 1000$ ), and $\beta$-actin (Santa Cruz; dilution, 1:5000). After being washed in Tris-buffered saline ( $\mathrm{pH} 7.2)$ containing $0.05 \%$ Tween 20 , the membranes were incubated with the secondary antibody for $2 \mathrm{~h}$ at room temperature. Blots were developed with electrochemiluminescence, and proteins were quantified by densitometry using $\beta$-actin as a loading control. Data from at least three independent experiments were collected for quantitative analysis.

2.10. mtDNA Copy Number. Total DNA was isolated from heart tissues and the relative mtDNA copy number was determined by qPCR as previously described [30]. The cytochrome $\mathrm{c}$-oxidase subunit I gene of the mitochondrial DNA (mtDNA) was quantified and normalized against NDUFV1 nDNA gene. The cytochrome c-oxidase subunit I primers were 5TGCTAGCCGCAGGCATTAC-3 (forward primer) and 5GGGTGCCCAAAGAATCAGAAC-3 (reverse primer). The NDUFV1 primers were 5-CTTCCCCACTGGCCTCAAG-3 (forward primer) and 5-CCAAAACCCAGTGATCCAGC-3 (reverse primer). qPCR was performed on multiplex realtime fluorescence quantitative PCR (iQ 5, BIORAD, USA) using PCR Supermix (Invitrogen). The relative mtDNA to nDNA ratio in each sample was determined based on amplification curves.

2.11. Statistical Analysis. Statistical analysis was performed using GraphPad Prism 5.0. Data were expressed as mean \pm standard error of at least three independent experiments. Comparison between groups was determined by one-way analysis of variance followed by paired $t$-test or $2 \times 2$ factorial design analysis. A level of $P<0.05$ was considered statistically significant.

\section{Results}

3.1. CVB-D Reduces DOX-Induced Cardiac Contractile Dysfunction and Histological Alterations. Numerous studies have shown that DOX may induce heart contractile dysfunction soon after administration $[2,31]$. In the present study, mouse heart function was monitored by echocardiography four days after a single administration of DOX. In our preliminary experiments, we have compared the echocardiographic data for each animal before/after CVB-D treatment. No significant difference was found between CVB-D group and vehicle group, and no obvious change was found after CVB-D administration compared to predose data (data not shown). Representative two-dimensional M-mode tracings of left ventricular wall motion were shown in Figure 1(a). Compared with the control, the range of waveforms was significantly decreased by DOX, and this change was reduced by pretreatment with CVB-D. By quantitative analysis of these echocardiograms, we found that CVB-D alone has no effect on heart function in physiological condition. DOX induced significant increases of $30.7 \%$ and $85.4 \%$, respectively, for LVEDD and LVEDS and decreases of $57.1 \%$ and $65.0 \%$, respectively, for ejection fraction and fractional shortening. Compared to DOX treated mice, mice treated with CVB-D plus DOX showed improved heart function as indicated by lower LVEDD and LVEDS and higher ejection fraction and fractional shortening. 

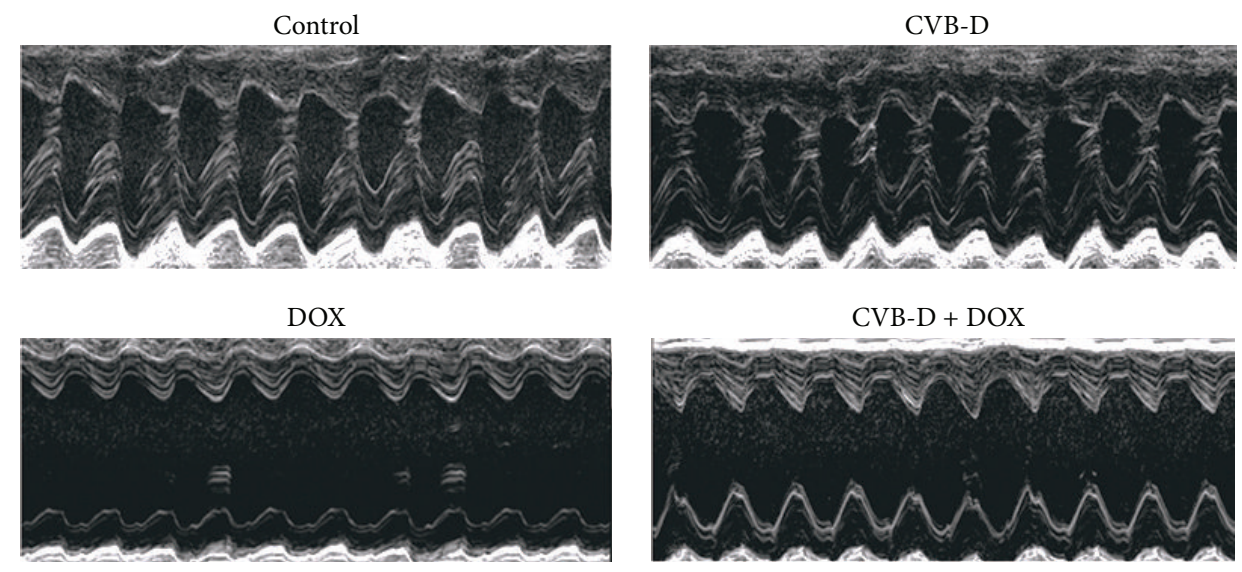

(a)

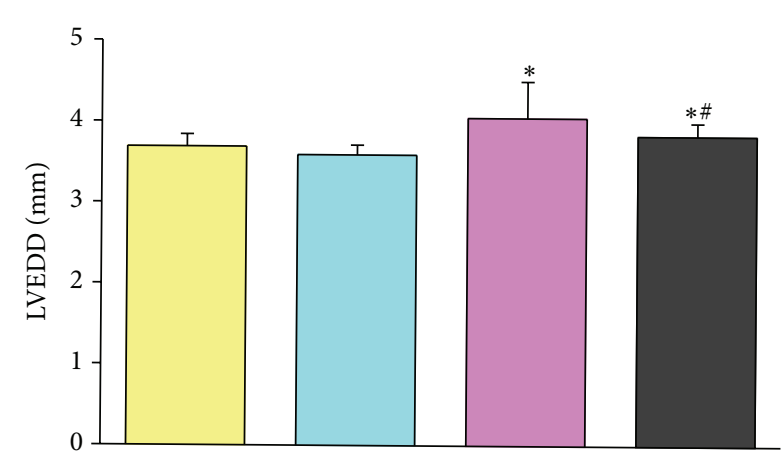

(b)

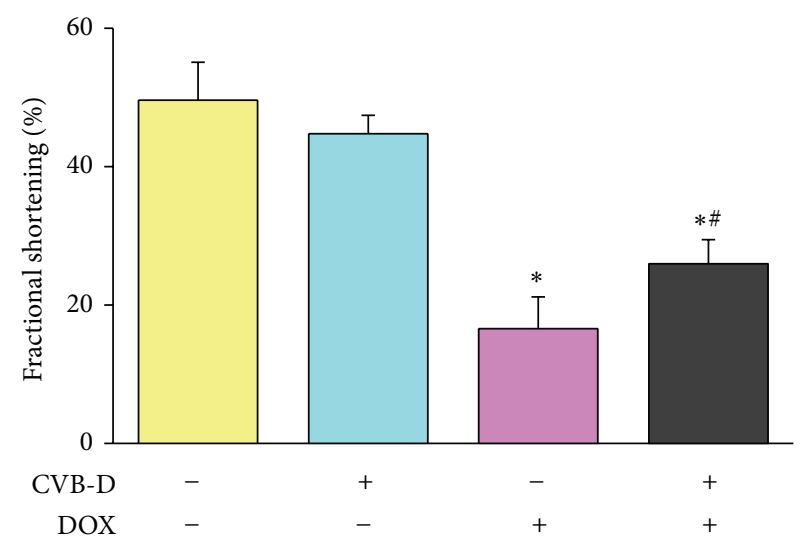

(d)

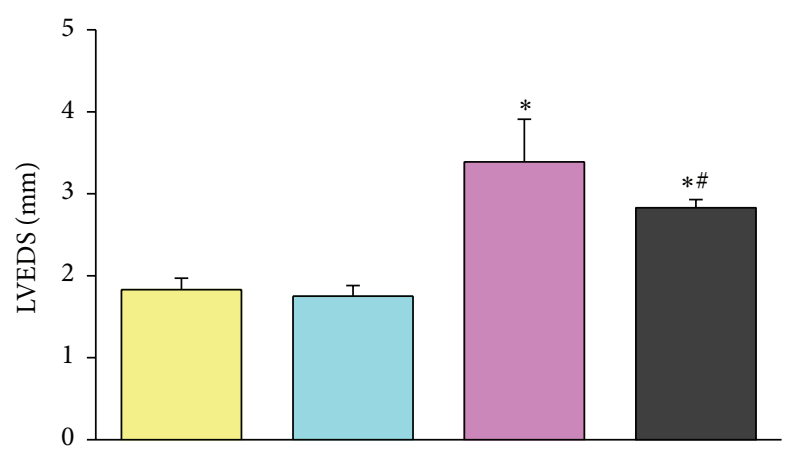

(c)

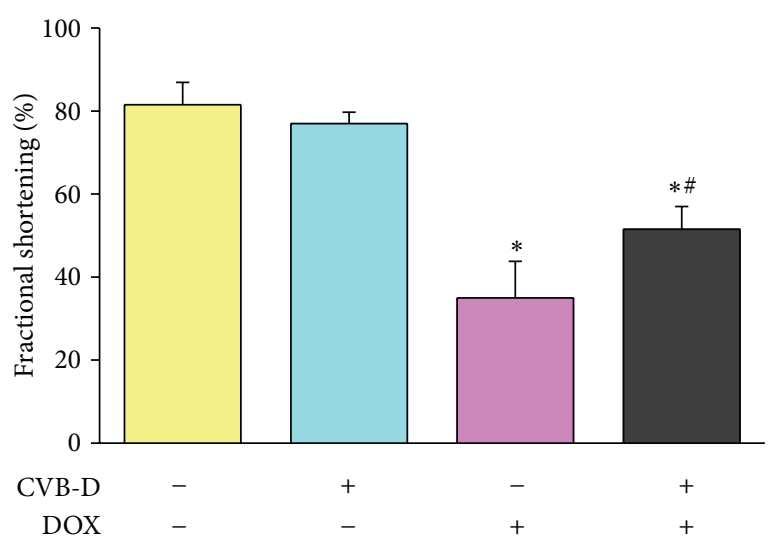

(e)

FIGURE 1: CVB-D attenuates DOX-induced cardiac contractile dysfunction. (a) Representative mouse short-axis echocardiograms. Cardiac function parameters were indicated by (b) LVEDD and (c) LVESD, (d) ejection fraction, and (e) fractional shortening. ${ }^{*} P<0.05$ versus the control group; ${ }^{\#} P<0.05$ versus the DOX group, $n=6$.

To demonstrate cardiac morphological alterations, sections of mouse heart tissue stained with hematoxylineosin were examined by light microscopy. As shown in Figure 2, heart section from control showed normal cardiac morphology. DOX induced obvious myocardial pathology including reduced myofibrils, swelling, vacuolization, and nuclear condensation or dissolution. CVB-D by itself had no effect on cardiac morphology; however, pretreatment with CVB-D significantly ameliorated DOX-induced lesions on myocardial morphology. DOX-induced cardiotoxicity has been shown to manifest similar pathophysiological characteristics with myocardial infarction-induced cardiac injury, such as heart dysfunction and morphology alterations. Thus, these findings are consistent with the recent studies 


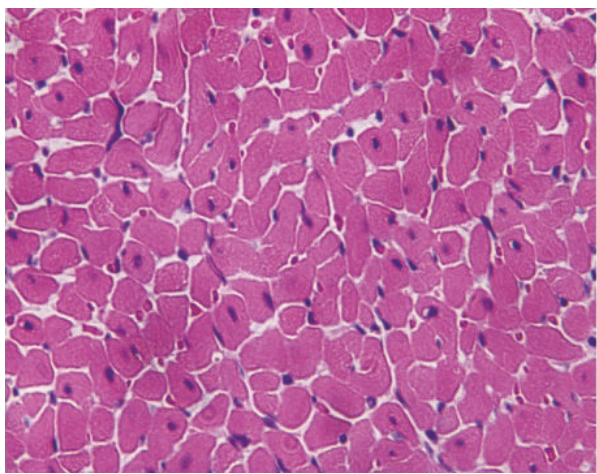

(a)

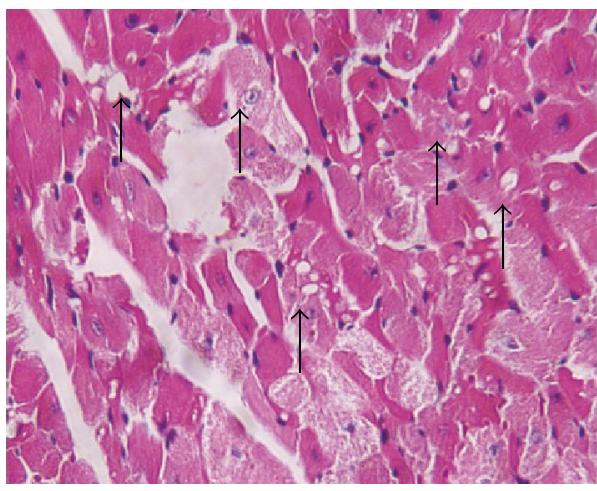

(c)

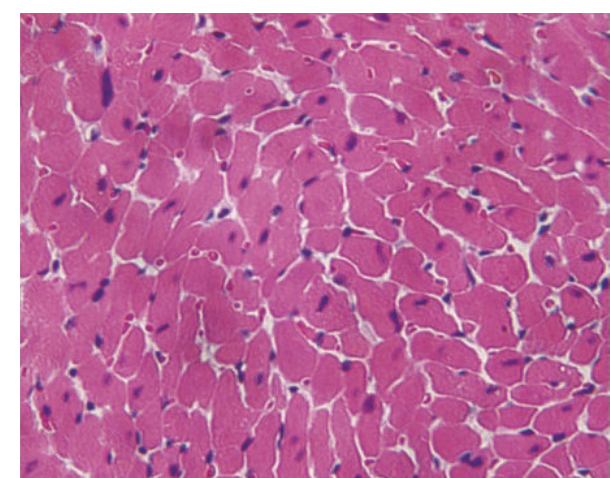

(b)

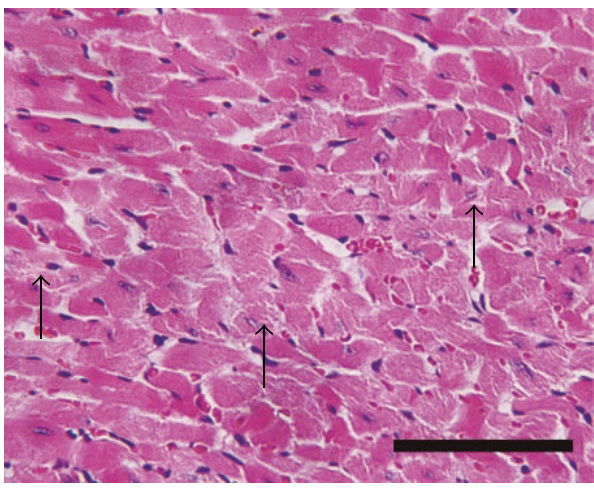

(d)

FIGURE 2: Light micrograph demonstrating the effect of CVB-D on DOX-induced myocardial histological alterations. Representative photomicrographs of mouse heart stained with H\&E. (a) Normal saline-treated control; (b) CVB-D pretreated group; (c) DOX-treated group; (d) CVB-D plus DOX-treated group. Arrows indicate areas of histological changes including reduced myofibrils, swelling, vacuolization, and nuclear condensation or dissolution. Scale bar $=20 \mu \mathrm{m}$.

which indicate that CVB-D provides efficient protection against myocardial infarction-induced cardiac dysfunction and pathological changes.

\subsection{CVB-D Inhibits DOX-Induced Myocardial Apoptosis and} Mitochondrial Cytochrome c Release. Apoptosis of cardiac cells has been suggested to be critically involved in the development of myocardial loss and severe contractile dysfunction during the pathogenesis of DOX cardiomyopathy $[10,32]$. By application of TUNEL assay, a significant increase of apoptotic cells was found in heart section from DOX group. Mice in CVB-D group and vehicle group showed similar apoptotic ratio which is under $1 \%$, indicating that CVB$\mathrm{D}$ had no effect on myocardial cell apoptosis in vivo. In contrast, CVB-D pretreatment effectively alleviated DOXinduced cardiac apoptosis. The apoptotic ratio was about $10.35 \%$ and 7.1\%, respectively, for DOX and CVB-D + DOX group (Figure 3).

Mitochondrial cytochrome $\mathrm{c}$ release into cytosol plays a pivotal role in DOX-induced apoptosis. As shown in Figure 4, western blot analysis revealed that DOX treatment significantly increased cytosolic concentrations of cytochrome $\mathrm{c}$ with a decrease in mitochondria. Pretreatment with CVB$\mathrm{D}$ preserved cytochrome $\mathrm{c}$ in mitochondria against DOX, as evidenced by significantly improved cytochrome $\mathrm{c}$ release into cytosol in CVB-D + DOX group. CVB-D alone had no effect on the distribution of cardiac cytochrome $c$ in mitochondria and cytosol. These findings are consistent with the results from TUNEL assay and correlate with cardiac function alterations.

3.3. CVB-D Ameliorates DOX-Induced Oxidative Damage. DOX-induced oxidative damage was evaluated by determination of lipid peroxidation and protein carbonylation. As shown in Figure 5, we did not observe significant difference in the level of TBARS in normal saline-treated mice and CVB-D treated mice. DOX treatment significantly increased cardiac TBARS level, and this effect was ameliorated by CVB-D pretreatment. As compared to that in normal salinetreated mice, the cardiac TBARS level in DOX-treated mice and CVB-D + DOX-treated animals was increased by $68.0 \%$ and $51.4 \%$, respectively (Figure $4(a)$ ). Similar results were found in the assessment of cardiac protein oxidative injury. CVB-D by itself had no effect on protein carbonylation but significantly inhibited DOX-induced protein carbonyl accumulation. The cardiac protein carbonyl contents were found to be increased by 13.0- and 10.2-fold, respectively, for DOX-treated mice and CVB-D + DOX-treated animals compared to that in controlled mice (Figure 4(b)). 


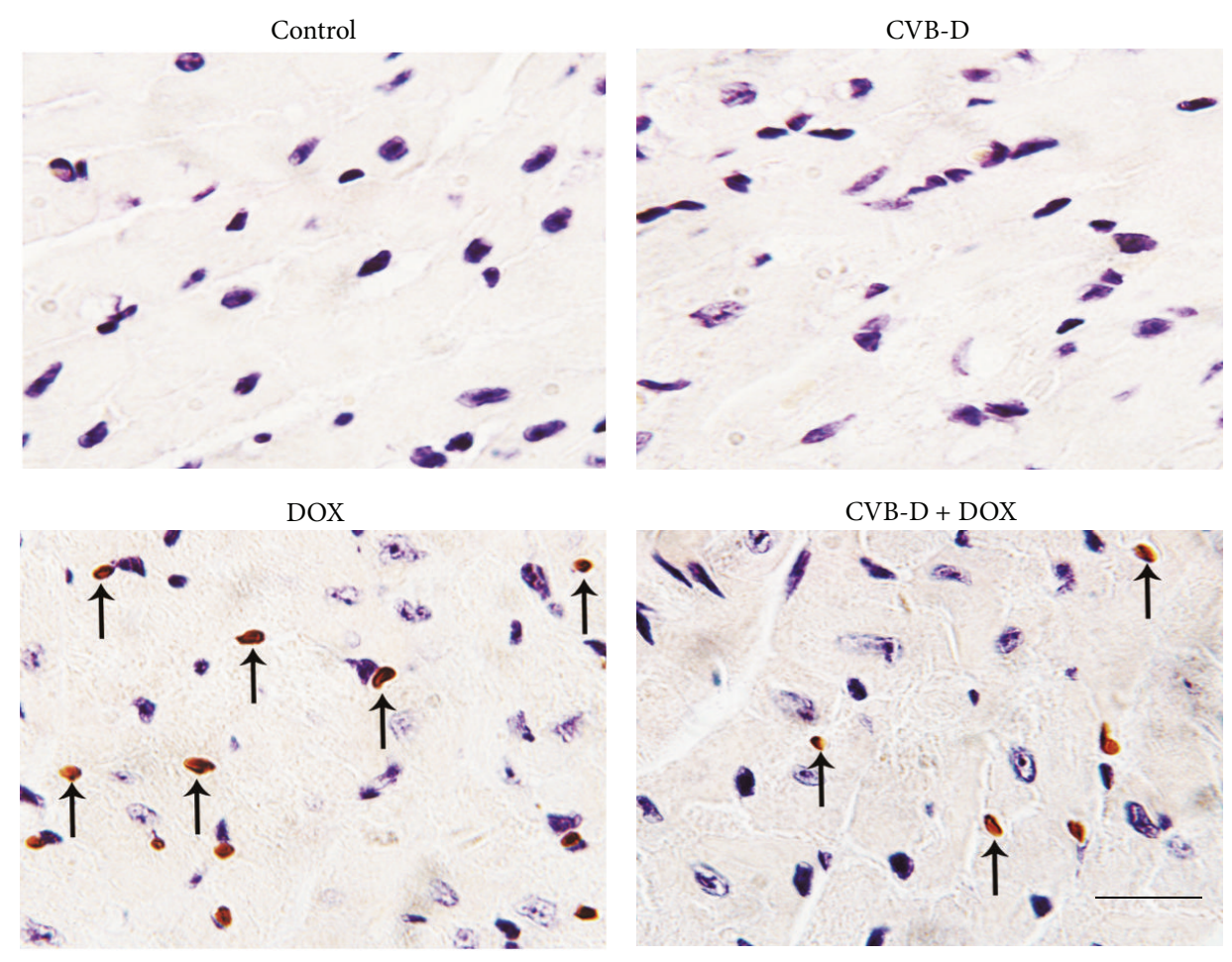

(a)

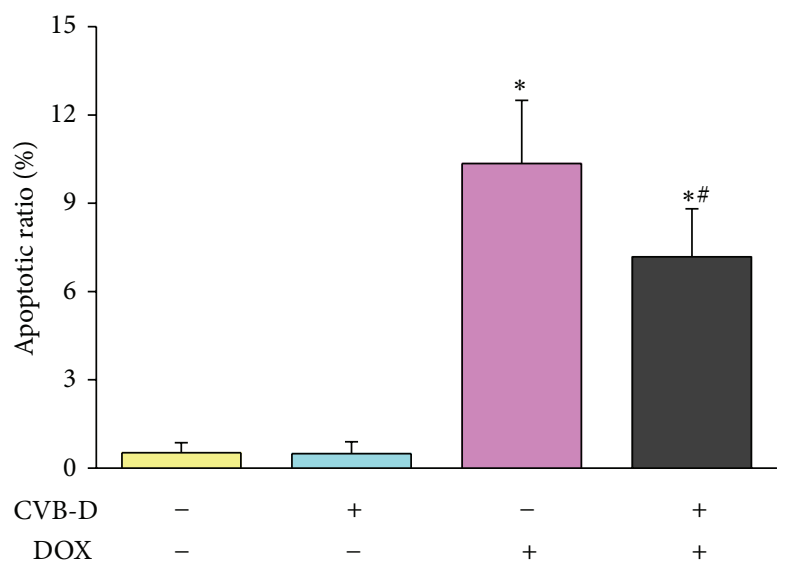

(b)

FIGURE 3: CVB-D relieves DOX-induced apoptosis in cardiac cells. Representative photomicrographs of TUNEL stained heart section. (a) Arrows indicate TUNEL-positive cells, scale bar $=20 \mu \mathrm{m}$. (b) Quantitative analysis of the percentage of apoptotic cells.

The antioxidant defense system is an important factor determining the fate of DOX-induced oxidative injury [5, 31, 33]. As one of the most important antioxidants in the heart, GSH plays a key role in the detoxification of a variety of chemicals including DOX [34]. Maintaining an optimal GSH/GSSG ratio is critical to the survival of cardiac cells and proper heart function. The ratio of GSH to GSSG has been widely used as a marker for oxidative stress in the cardiovascular system $[35,36]$. In the present study, GSH/GSSG ratio was decreased by $43.0 \%$ in DOX-treated mice as compared to normal saline-treated mice. CVB-D by itself did not affect the ratio of GSH/GSSG in the heart under physiological condition. Pretreatment with CVB-D significantly prevented
DOX-induced reduction of GSH/GSSG ratio. The cardiac GSH/GSSG ratio in CVB-D + DOX-treated mice was 1.26fold higher than that in DOX-treated mice (Figure 6).

3.4. CVB-D Improves Cardiac Mitochondrial Biogenesis Impairments by DOX. A growing body of evidence suggests that impaired cardiac mitochondrial biogenesis plays a key role in DOX cardiotoxicity [37-39]. PGC- $1 \alpha$ is emerged as a master regulator for mitochondrial biogenesis. PGC- $1 \alpha$ interacts with NRFs, to activate mitochondrial biogenesis by facilitating transcription, translation, and activation of downstream transcription factors necessary for mtDNA replication [40]. In the present study, the expression of PGC- $1 \alpha$ and NRF-1 


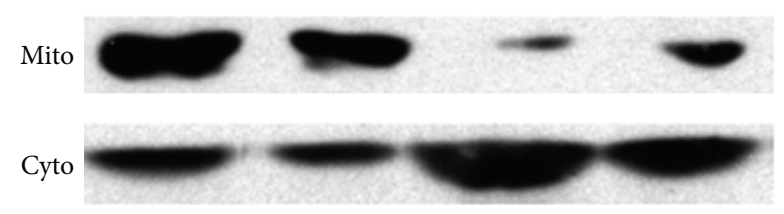

(a)

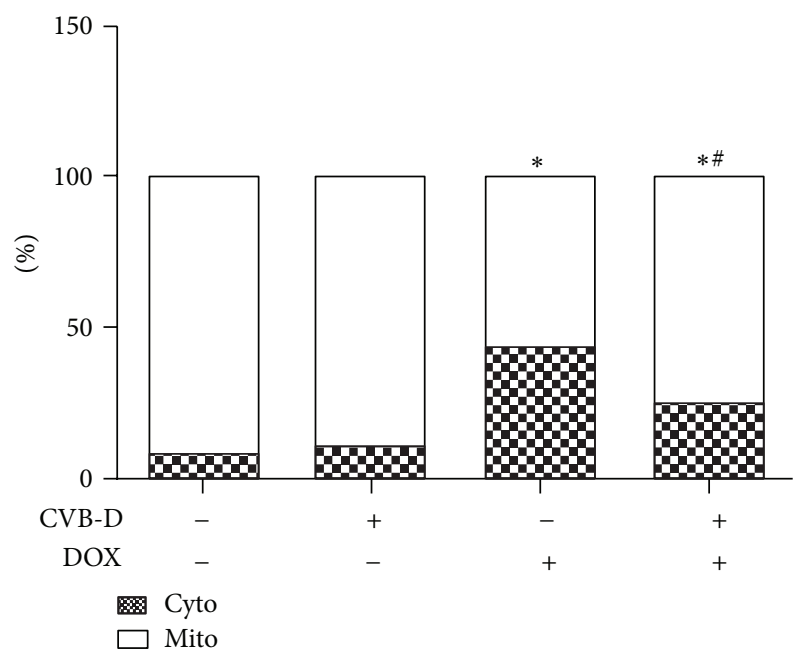

(b)

FIGURE 4: CVB-D inhibits cytochrome $\mathrm{c}$ release from mitochondria into cytosol in the heart. (a) Representative blots of cytochrome $\mathrm{c}$ in cardiac mitochondrial and cytosolic fractions. (b) Quantitative analysis of cytochrome $\mathrm{c}$ protein expression. ${ }^{*} P<0.05$ versus the control group; ${ }^{\#} P<0.05$ versus the DOX group.

as well as mtDNA copy number was determined to evaluate the effect of CVB-D on DOX-induced cardiac mitochondrial biogenesis impairment. As shown in Figure 7(a), CVB-D pretreatment has no obvious effect on the protein expression of cardiac PGC- $1 \alpha$ and NRF-1. However, CVB-D was found to effectively improve DOX-induced decreased expression of both PGC- $1 \alpha$ and NRF-1. These changes were well mirrored by the results from the assay of mtDNA copy number, one of the most frequently used markers for mitochondrial biogenesis. As compared to the mice in control group, cardiac mtDNA copy number of DOX-treated mice was decreased by $78.8 \%$, while mice treated with CVB-D plus DOX showed a significant ameliorated mtDNA injury with a reduction of 54.0\% (Figure 7(b)).

\section{Discussion}

DOX has been used in chemotherapy as one of the most effective anticancer agents since the late 1960s. Despite its efficacy, the application of DOX is greatly compromised by its cardiotoxicity. Significant efforts have been made in the past decades aiming at prevention and/or attenuation of DOX-induced cardiotoxicity, but, so far, the efficiency of these interventions is limited $[1,5,41]$. The present study showed for the first time that CVB-D pretreatment

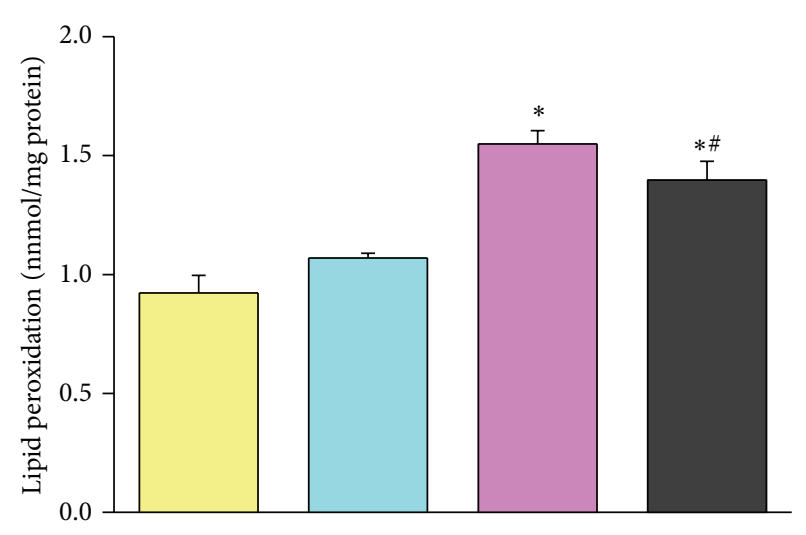

(a)

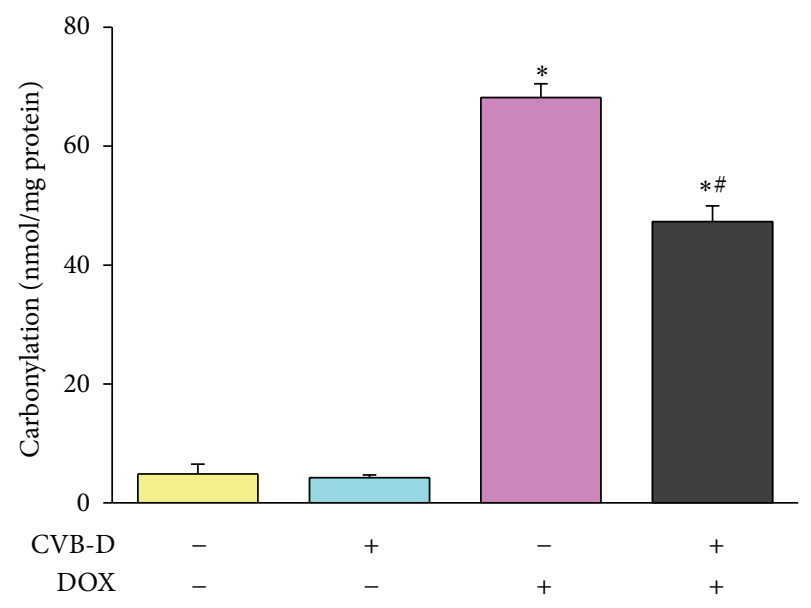

(b)

FIGURE 5: CVB-D protects against DOX-induced cardiac oxidative damage indicated by (a) lipid peroxidation and (b) protein carbonylation. ${ }^{*} P<0.05$ versus the control group; ${ }^{\#} P<0.05$ versus the DOX group, $n=6$.

significantly improved DOX-induced cardiac contractile dysfunction, histological alterations, apoptosis, and mitochondrial cytochrome $c$ release to cytosol. Pretreatment with CVB-D ameliorated DOX-induced cardiac oxidative damage including lipid peroxidation and protein carbonylation and a decrease in GSH to GSSG ratio. Moreover, CVB-D was found to prevent DOX-induced mitochondrial biogenesis impairment as indicated by preservation of PGC- $1 \alpha$ and NRF-1, as well as mitochondrial DNA copy number. These findings demonstrate that CVB-D protects against DOXinduced cardiomyopathy, at least in part, by suppression of oxidative damage and mitochondrial biogenesis impairment.

CVB-D has been increasingly shown to provide efficient protection against many cardiovascular diseases including myocardial infarction-induced heart failure $[14,18,19]$. By using primary cultured neonatal rat cardiomyocytes, $\mathrm{Hu}$ and colleagues previously showed that CVB-D pretreatment can reduce DOX-induced decrease of cell viability [23]. However, because of the much more complicated environment in in vivo where systemically metabolic, hormonal, and neuronal influences as well as compensatory effects cannot 


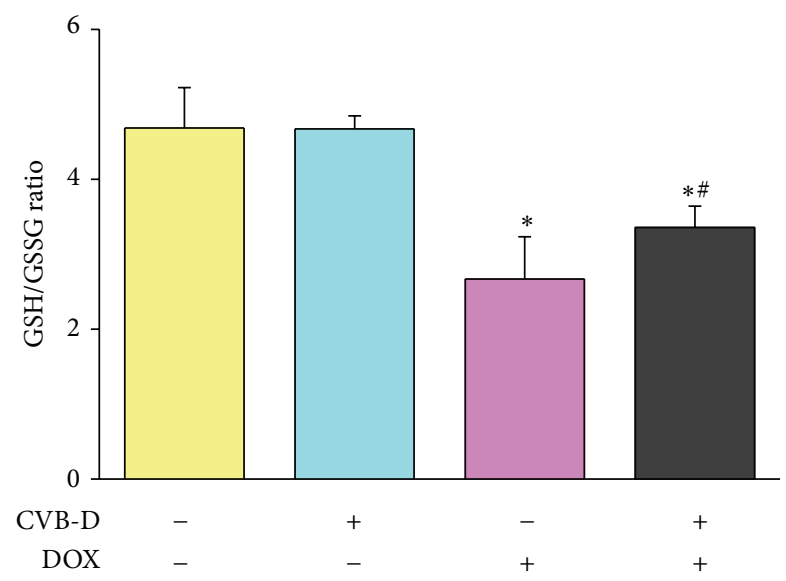

FIGURE 6: CVB-D alleviates DOX-induced decrease of GSH/GSSG ratio. ${ }^{*} P<0.05$ versus the control group; ${ }^{\#} P<0.05$ versus the DOX group, $n=6$.

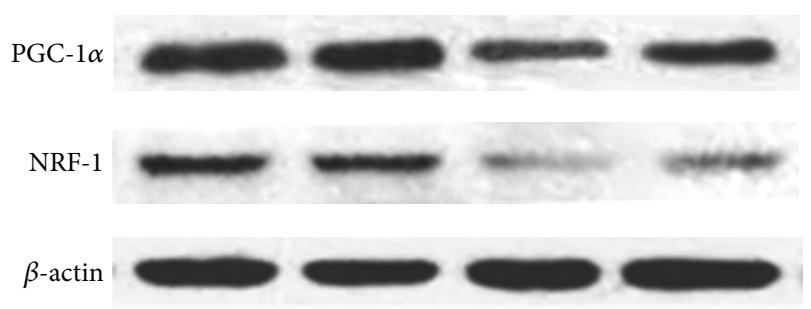

(a)

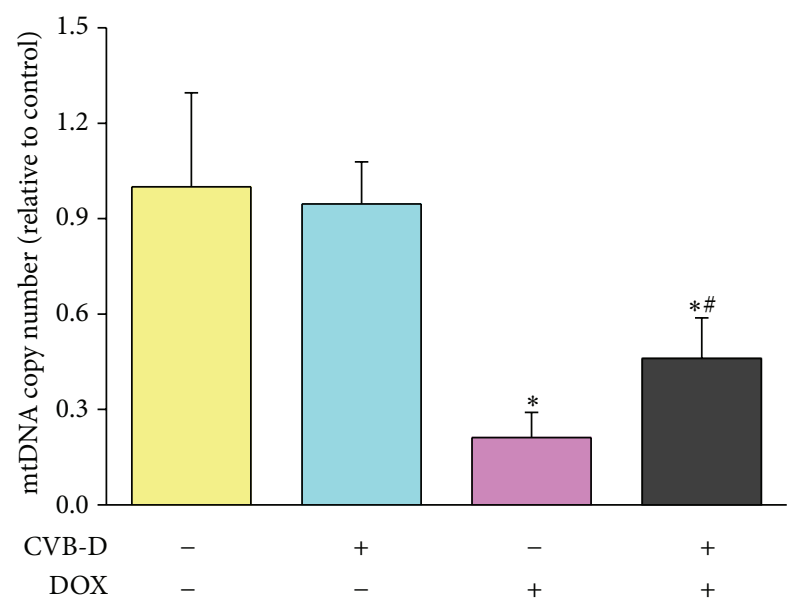

(b)

FIGURE 7: CVB-D ameliorates DOX-induced mitochondrial biogenesis impairment. (a) Representative blots of cardiac PGC- $1 \alpha$ and NRF-1; (b) mtDNA copy number determined by qPCR. ${ }^{*} P<0.05$ versus the control group; ${ }^{\#} P<0.05$ versus the DOX group.

be excluded, it is necessary to testify whether CVB-D is effective in protecting against DOX cardiotoxicity in vivo. Our results showed that CVB-D by itself has no obvious effects on cardiac function which is consistent with previous finding [42]; however, mice pretreated with CVB-D were well protected from DOX-induced cardiomyopathy as evidenced by significant improved cardiac contractile dysfunction, histological alterations, and apoptosis. DOX-induced cardiomyopathy is a complex multifactorial process that leads to heart damage and dysfunction, which is mediated by a series of events involving production of reactive oxygen species, disruption of mitochondrial biogenesis, energy depletion, alteration of ionic homeostasis, and cell death by apoptosis. These characteristics of DOX-induced are highly similar to that observed in ischemic heart failure $[1,7]$. Thus, our findings are in agreement with the previous studies showing that CVB-D protects the heart from myocardial infarctioninduced injuries $[18,23]$. Though no obvious changes by CVB-D was found under physiological conditions, CVB$\mathrm{D}$ may somewhat induce effects that are beneficial for the cardiovascular system to be protected from damage. Another possible explanation is that CVB-D may play some role in maintaining the homeostasis and preservation of cardiac function and exerts protective effects under pathological condition.

Cardiac cells contain abundant mitochondria which have been implicated to play a central role in the pathogenesis of cardiac injury [43]. DOX has a high affinity for cardiolipin which localizes in the inner membrane of mitochondria, leading to specific accumulation of DOX in cardiac mitochondria $[7,44]$. Increasing evidence suggests that DOX cardiotoxicity is mainly mediated by reactive oxygen species generation within mitochondria. During the metabolism, DOX undergoes one-electron reduction that is catalyzed by $\mathrm{NAD}(\mathrm{P}) \mathrm{H}$ reductases to yield a semiquinone free-radical intermediate, which regenerates its parent quinone by reacting with $\mathrm{O}_{2}$ to produce superoxide anion and other forms of reactive oxygen species [8]. As a main site for bioreductive metabolism of DOX and reactive oxygen species generation, mitochondria are also preferential targets of reactive oxygen species. Excess reactive oxygen species can attack the components of mitochondria, to induce mitochondrial dysfunction leading to oxidative damage, and ultimately initiate cell death such as apoptosis $[44,45]$. Our results showed that CVB-D pretreatment significantly improved DOX-induced cardiac oxidative damage as evidenced by ameliorated lipid peroxidation and protein carbonylation. These results are in agreement with previous report showing a lipid peroxidation inhibitory activity of CVB-D and its derivatives [24]. In addition, our results showed that CVB-D protected GSH, a predominant antioxidant in detoxification of DOX-induced oxidative stress. Because reactive oxygen species are very active and are easily metabolized, it is particularly not easy to precisely detect the level of reactive oxygen species in in vivo studies. Although our findings are not direct evidence demonstrating the effect of CVB-D on reactive oxygen species generation, they strongly suggest that CVB-D protect the heart from DOX toxicity by suppressing oxidative damage and preserving antioxidant capacity.

Mitochondria in cardiac cells are highly dynamic involving mitochondrial biogenesis which is crucial for energetic metabolism and maintaining a proper function of heart [ 40 , $46,47]$. In particular, mitochondrial biogenesis is important for overcoming mitochondrial damage and repairing cell injury under pathological conditions $[40,46]$. DOX has been 
shown to inhibit PGC- $1 \alpha$, a master regulator of mitochondrial biogenesis, and affect the transcription of mitochondrial genome, consequently leading to disruption of mitochondrial biogenesis $[10,37]$. Targeting at preservation of PGC- $1 \alpha$ and mitochondrial biogenesis by many pharmacological agents, including traditional Chinese medicines like QishenYiQi pills, has been shown to provide efficient protection against DOX-induced cardiac injuries [31, 48, 49]. Our results showed that, under physiological conditions, CVB-D neither affects the protein expression of PGC- $1 \alpha$ nor alters the cardiac mtDNA content. However, CVB-D pretreatment remarkably inhibited DOX-induced depression of PGC- $1 \alpha$ and NRF-1 protein expression. Consistent with these findings, CVB-D was also found to effectively prevent the decrease of mtDNA number by DOX, suggesting that the cardioprotective effect of CVB-D is associated with preservation of mitochondrial biogenesis.

In conclusion, the present study depicts a novel function of CVB-D in protection against DOX-induced cardiotoxicity and suggests that the cardioprotective action of CVB-D is mediated, at least in part, by suppression of oxidative damage and mitochondrial biogenesis impairment. Given the facts that CVB-D has been proven as an efficient protective agent against cardiovascular diseases like heart failure and lack of preventive or therapeutic options for the toxic cardiomyopathy of DOX, our data indicate that CVB-D may clinically provide a promising cardioprotection against DOX cardiotoxicity. However, many critical questions need further investigations. For example, pharmacokinetics of CVB-D in the current study is unrevealed. It is not clear whether CVB-D affects the antitumor activity of DOX. Future studies aiming at these questions will be important and informative for the clinical application of CVB-D in protecting patient from DOX-induced cardiac side effects during chemotherapy.

\section{Abbreviations}

CVB-D: Cyclovirobuxine D

DOX: Doxorubicin

DTNB: 5,5'-Dithio-2-nitrobenzoic acid

GSH: Reduced glutathione

GSSG: Oxidized glutathione

LVEDD: Left ventricular end-diastolic diameter

LVEDS: Left ventricular end-systolic diameter

mtDNA: Mitochondrial DNA

NRF-1: Nuclear respiratory factor 1

PGC-1 $\alpha$ : Peroxisome proliferators-activated receptor $\gamma$ coactivator $-1 \alpha$

TBARS: Thiobarbituric acid reactive substances

TUNEL: Terminal deoxynucleotidyl transferase-mediated dUTP nick end labeling.

\section{Conflict of Interests}

The authors declare that there is no conflict of interests.

\section{Authors' Contribution}

Qian Guo and Jiabin Guo have equally contributed to this work.

\section{Acknowledgments}

This project was supported the National Natural Science Foundation of China (81470167, 81430090, and 81072711) and National Key Project on Drug Development from the Ministry of Science and Technology of China (2012ZX09J12203) and partly financed by the State Key Laboratory of Environmental Chemistry and Ecotoxicology, Research Center for Eco-Environmental Sciences, Chinese Academy of Sciences (KF2011-04).

\section{References}

[1] E. Hao, P. Mukhopadhyay, Z. Cao et al., "Cannabidiol protects against doxorubicin-induced cardiomyopathy by modulating mitochondrial function and biogenesis," Molecular Mecicine, 2015.

[2] M. S. Ewer and S. M. Ewer, "Cardiotoxicity of anticancer treatments: what the cardiologist needs to know," Nature Reviews Cardiology, vol. 7, no. 10, pp. 564-575, 2010.

[3] M. Lotrionte, G. Biondi-Zoccai, A. Abbate et al., "Review and meta-analysis of incidence and clinical predictors of anthracycline cardiotoxicity," The American Journal of Cardiology, vol. 112, no. 12, pp. 1980-1984, 2013.

[4] P. K. Singal and N. Iliskovic, "Doxorubicin-induced cardiomyopathy," The New England Journal of Medicine, vol. 339, no. 13, pp. 900-905, 1998.

[5] Y. Octavia, C. G. Tocchetti, K. L. Gabrielson, S. Janssens, H. J. Crijns, and A. L. Moens, "Doxorubicin-induced cardiomyopathy: from molecular mechanisms to therapeutic strategies," Journal of Molecular and Cellular Cardiology, vol. 52, no. 6, pp. 1213-1225, 2012.

[6] D. Harake, V. I. Franco, J. M. Henkel, T. L. Miller, and S. E. Lipshultz, "Cardiotoxicity in childhood cancer survivors: strategies for prevention and management," Future Cardiology, vol. 8, no. 4, pp. 647-670, 2012.

[7] G. Takemura and H. Fujiwara, "Doxorubicin-induced cardiomyopathy from the cardiotoxic mechanisms to management," Progress in Cardiovascular Diseases, vol. 49, no. 5, pp. 330-352, 2007.

[8] K. J. A. Davies and J. H. Doroshow, "Redox cycling of anthracyclines by cardiac mitochondria. I. Anthracycline radical formation by NADH dehydrogenase," The Journal of Biological Chemistry, vol. 261, no. 7, pp. 3060-3067, 1986.

[9] E. A. Konorev, S. Vanamala, and B. Kalyanaraman, "Differences in doxorubicin-induced apoptotic signaling in adult and immature cardiomyocytes," Free Radical Biology and Medicine, vol. 45, no. 12, pp. 1723-1728, 2008.

[10] H. B. Suliman, M. S. Carraway, A. S. Ali, C. M. Reynolds, K. E. Welty-Wolf, and C. A. Piantadosi, "The CO/HO system reverses inhibition of mitochondrial biogenesis and prevents murine doxorubicin cardiomyopathy," The Journal of Clinical Investigation, vol. 117, no. 12, pp. 3730-3741, 2007.

[11] N. A. E. Boghdady, "Antioxidant and antiapoptotic effects of proanthocyanidin and ginkgo biloba extract against doxorubicin-induced cardiac injury in rats," Cell Biochemistry and Function, vol. 31, no. 4, pp. 344-351, 2013. 
[12] O. A. Alshabanah, M. M. Hafez, M. M. Al-Harbi et al., "Doxorubicin toxicity can be ameliorated during antioxidant L-carnitine supplementation," Oxidative Medicine and Cellular Longevity, vol. 3, no. 6, pp. 428-433, 2010.

[13] L. Chaiswing, M. P. Cole, W. Ittarat, L. I. Szweda, D. K. St. Clair, and T. D. Oberley, "Manganese superoxide dismutase and inducible nitric oxide synthase modify early oxidative events in acute Adriamycin-induced mitochondrial toxicity," Molecular Cancer Therapeutics, vol. 4, no. 7, pp. 1056-1064, 2005.

[14] Q.-W. Chen, H.-L. Shan, H.-L. Sun, H. Wang, and B.-F. Yang, "Effects of cyclovirobuxine $\mathrm{D}$ on intracellular $\mathrm{Ca}^{2+}$ and $\mathrm{L}$ type $\mathrm{Ca}^{2+}$ current in rat ventricular cardiomyocytes," Acta Pharmaceutica Sinica, vol. 39, no. 7, pp. 500-503, 2004.

[15] M. Yan, "Congestive heart failure treated by a combination of cyclovirobuxine D with digoxin," Zhong Xi Yi Jie He Za Zhi, vol. 10, no. 2, pp. 88-89, 1990.

[16] Y. X. Wang, J. W. Liu, Y. H. Tau, and B. H. Sheng, "Antiarrhythmic action of cycloprotobuxine-A," Acta Pharmacologica Sinica, vol. 10, no. 5, pp. 389-393, 1989.

[17] P. Shan, R. B. Mao, J. M. Xu, and J. X. Li, "The beneficial effects of cyclovirobuxine D (CVBD) in coronary heart disease. A double blind analysis of 110 cases.," Journal of Traditional Chinese Medicine, vol. 4, no. 1, pp. 15-19, 1984.

[18] B. Yu, T.-H. Fang, G.-H. Lü, H.-Q. Xu, and J.-F. Lu, "Beneficial effect of Cyclovirobuxine D on heart failure rats following myocardial infarction," Fitoterapia, vol. 82, no. 6, pp. 868-877, 2011.

[19] E. Grossini, A. Battaglia, S. Brunelleschi et al., "Coronary effects of cyclovirobuxine $\mathrm{D}$ in anesthetized pigs and in isolated porcine coronary arteries," Life Sciences, vol. 65, no. 5, pp. PL59PL65, 1999.

[20] "Double blind study of the therapeutic effect of cyclovirobuxine D No. 1 on the left ventricular function in coronary heart disease," Zhonghua Xin Xue Guan Bing Za Zhi, vol. 11, pp. 89-91, 1983.

[21] X. J. Liu, M. H. Yao, T. H. Fang, Y. Y. Song, B. Y. Wu, and R. Y. Sun, "Some cardiovascular effects of cyclovirobuxine-D," Acta Pharmacologica Sinica, vol. 3, no. 2, pp. 101-104, 1982.

[22] E. Grossini, G. Avanzi, M. Gallicchio, C. Molinari, G. Vacca, and G. Bellomo, "Regulation of $\mathrm{Ca}^{2+}$ movements by cyclovirobuxine D in ECV304 endothelial cells," Pharmacological Research, vol. 52, no. 2, pp. 154-161, 2005.

[23] D. Hu, X. Liu, Y. Wang, and S. Chen, "Cyclovirobuxine D ameliorates acute myocardial ischemia by $K_{A T P}$ channel opening, nitric oxide release and anti-thrombosis," European Journal of Pharmacology, vol. 569, no. 1-2, pp. 103-109, 2007.

[24] L. Deng, H. Huang, and M.-X. Xu, "Structural modification of cyclovirobuxine D and their inhibition activity on lipid peroxidation," Acta pharmaceutica Sinica, vol. 41, no. 2, pp. 121-124, 2006.

[25] F.-F. Yang and S.-J. Hu, "Effect of cyclovirobuxine-D on heart function and blood pressure of rats in vivo," Chinese Journal of Pharmacology and Toxicology, vol. 21, no. 4, pp. 255-258, 2007.

[26] P. Yu, W. Y. Liu, S. Gao et al., "Pharmacokinetic study of clovirobuxine D in healthy volunteers," Chinese Pharmaceutical Journal, vol. 43, no. 3, pp. 203-207, 2008.

[27] A. Razmara, L. Sunday, C. Stirone et al., "Mitochondrial effects of estrogen are mediated by estrogen receptor $\alpha$ in brain endothelial cells," Journal of Pharmacology and Experimental Therapeutics, vol. 325, no. 3, pp. 782-790, 2008.
[28] X. Yu, J. Guo, H. Fang, and S. Peng, "Basal metallothionein-I/II protects against NMDA-mediated oxidative injury in cortical neuron/astrocyte cultures," Toxicology, vol. 282, no. 1-2, pp. 1622, 2011.

[29] E. Floor and M. G. Wetzel, "Increased protein oxidation in human substantia nigra pars compacta in comparison with basal ganglia and prefrontal cortex measured with an improved dinitrophenylhydrazine assay," Journal of Neurochemistry, vol. 70, no. 1, pp. 268-275, 1998.

[30] W. Guo, L. Jiang, S. Bhasin, S. M. Khan, and R. H. Swerdlow, "DNA extraction procedures meaningfully influence qPCRbased mtDNA copy number determination," Mitochondrion, vol. 9, no. 4, pp. 261-265, 2009.

[31] S.-G. Zhu, R. C. Kukreja, A. Das, Q. Chen, E. J. Lesnefsky, and $\mathrm{L}$. Xi, "Dietary nitrate supplementation protects against doxorubicin-induced cardiomyopathy by improving mitochondrial function," Journal of the American College of Cardiology, vol. 57, no. 21, pp. 2181-2189, 2011.

[32] X.-L. Wang, L.-L. Xiong, Y. Zhu et al., "Salidroside improves doxorubicin-induced cardiac dysfunction by suppression of excessive oxidative stress and cardiomyocyte apoptosis," Journal of Cardiovascular Pharmacology, vol. 62, no. 6, pp. 512-523, 2013.

[33] Z. Fu, J. Guo, L. Jing, R. Li, T. Zhang, and S. Peng, "Enhanced toxicity and ROS generation by doxorubicin in primary cultures of cardiomyocytes from neonatal metallothionein-I/II null mice," Toxicology in Vitro, vol. 24, no. 6, pp. 1584-1591, 2010.

[34] G. Singh, A. T. Singh, A. Abraham et al., "Protective effects of Terminalia arjuna against Doxorubicin-induced cardiotoxicity," Journal of Ethnopharmacology, vol. 117, no. 1, pp. 123-129, 2008.

[35] R. Asmis, Y. Wang, L. Xu, M. Kisgati, J. G. Begley, and J. J. Mieyal, "A novel thiol oxidation-based mechanism for adriamycin-induced cell injury in human macrophages," The FASEB Journal, vol. 19, no. 13, pp. 1866-1868, 2005.

[36] A. Ozkan and K. Fişkin, "Epirubicin $\mathrm{HCl}$ toxicity in humanliver derived hepatoma G2 cells," Polish Journal of Pharmacology, vol. 56, no. 4, pp. 435-444, 2004.

[37] S. Zhang, X. Liu, T. Bawa-Khalfe et al., "Identification of the molecular basis of doxorubicin-induced cardiotoxicity," Nature Medicine, vol. 18, no. 11, pp. 1639-1642, 2012.

[38] E. Jirkovsky, O. Popelová, P. Kriváková-Stanková et al., “Chronic anthracycline cardiotoxicity: molecular and functional analysis with focus on nuclear factor erythroid 2-related factor 2 and mitochondrial biogenesis pathways," Journal of Pharmacology and Experimental Therapeutics, vol. 343, no. 2, pp. 468-478, 2012.

[39] E. Sahin, S. Colla, M. Liesa et al., "Telomere dysfunction induces metabolic and mitochondrial compromise," Nature, vol. 470, no. 7334, pp. 359-365, 2011.

[40] I. S. Patten and Z. Arany, "PGC-1 coactivators in the cardiovascular system," Trends in Endocrinology and Metabolism, vol. 23, no. 2, pp. 90-97, 2012.

[41] M. Štěrba, O. Popelová, A. Vávrová et al., "Oxidative stress, redox signaling, and metal chelation in anthracycline cardiotoxicity and pharmacological cardioprotection," Antioxidants and Redox Signaling, vol. 18, no. 8, pp. 899-929, 2013.

[42] Z. Q. Chen, S. J. Hu, W. Y. Shi, J. Du, Y. Shen, and Q. Xia, "Electrophysiologic study of the biphasic effects of cyclovirobuxine D on arrhythmias," Chinese Journal of Integrated Traditional and Western Medicine, vol. 24, pp. 1010-1013, 2004. 
[43] M. Bayeva, M. Gheorghiade, and H. Ardehali, "Mitochondria as a therapeutic target in heart failure," Journal of the American College of Cardiology, vol. 61, no. 6, pp. 599-610, 2013.

[44] J. M. Berthiaume and K. B. Wallace, "Adriamycin-induced oxidative mitochondrial cardiotoxicity," Cell Biology and Toxicology, vol. 23, no. 1, pp. 15-25, 2007.

[45] K. Brieger, S. Schiavone, F. J. Miller Jr., and K.-H. Krause, "Reactive oxygen species: from health to disease," Swiss Medical Weekly, vol. 142, Article ID w13659, 2012.

[46] J. Ren, L. Pulakat, A. Whaley-Connell, and J. R. Sowers, "Mitochondrial biogenesis in the metabolic syndrome and cardiovascular disease," Journal of Molecular Medicine, vol. 88, no. 10, pp. 993-1001, 2010.

[47] E. Nisoli, E. Clementi, M. O. Carruba, and S. Moncada, "Defective mitochondrial biogenesis: a hallmark of the high cardiovascular risk in the metabolic syndrome?" Circulation Research, vol. 100, no. 6, pp. 795-806, 2007.

[48] D.-X. Tang, H.-P. Zhao, C.-S. Pan et al., "QiShenYiQi pills, a compound chinese medicine, ameliorates doxorubicin-induced myocardial structure damage and cardiac dysfunction in rats," Evidence-based Complementary and Alternative Medicine, vol. 2013, Article ID 480597, 9 pages, 2013.

[49] X. Marechal, D. Montaigne, C. Marciniak et al., "Doxorubicininduced cardiac dysfunction is attenuated by ciclosporin treatment in mice through improvements in mitochondrial bioenergetics," Clinical Science, vol. 121, no. 9, pp. 405-413, 2011. 


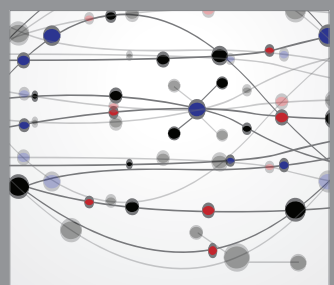

The Scientific World Journal
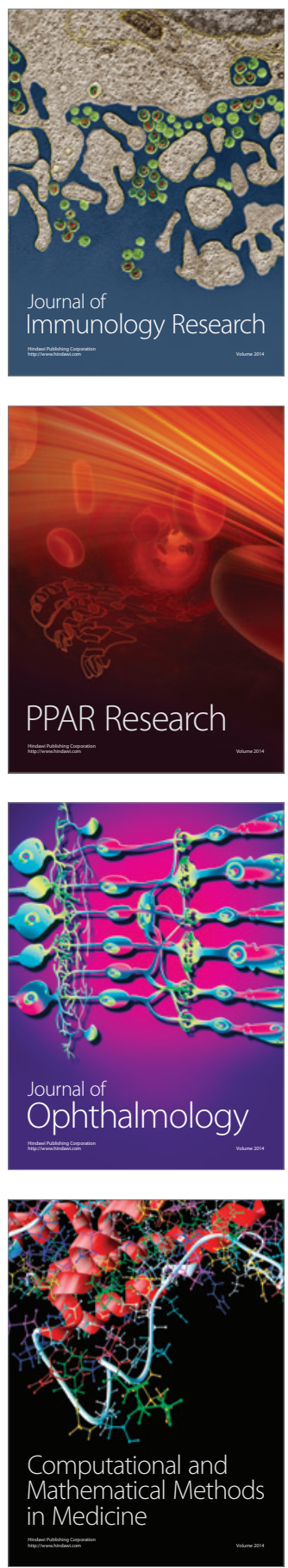

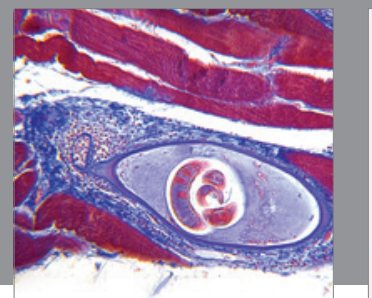

Gastroenterology

Research and Practice
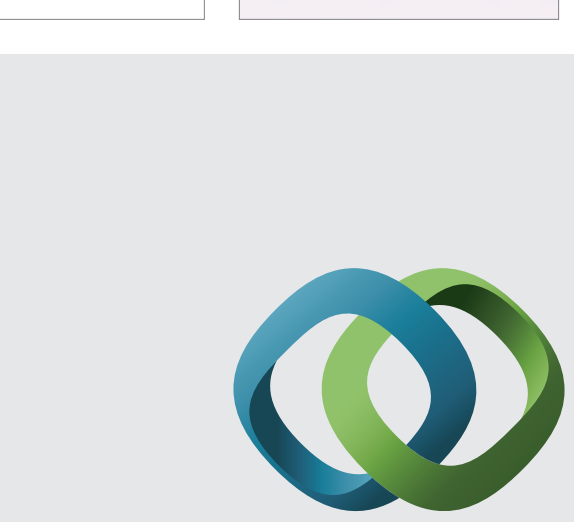

\section{Hindawi}

Submit your manuscripts at

http://www.hindawi.com
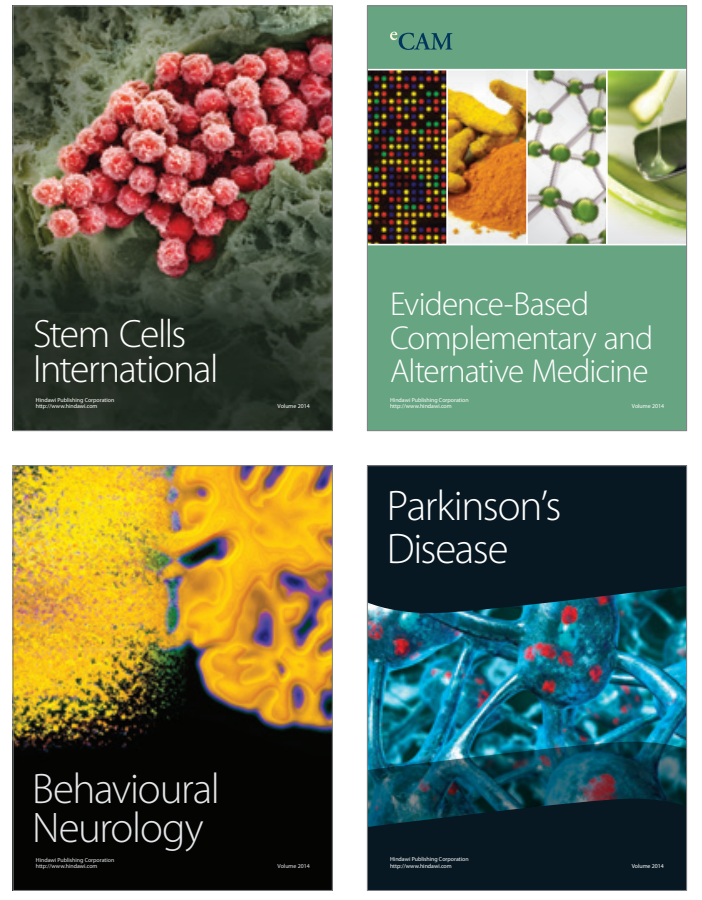
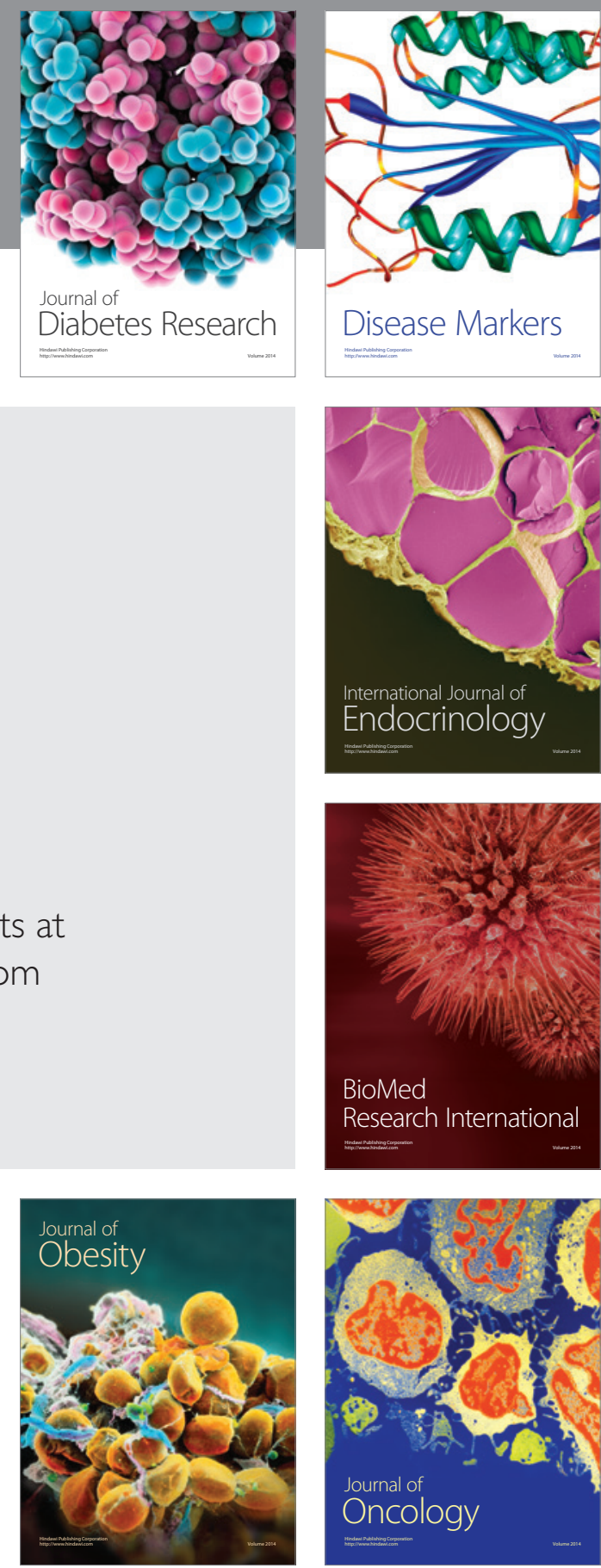

Disease Markers
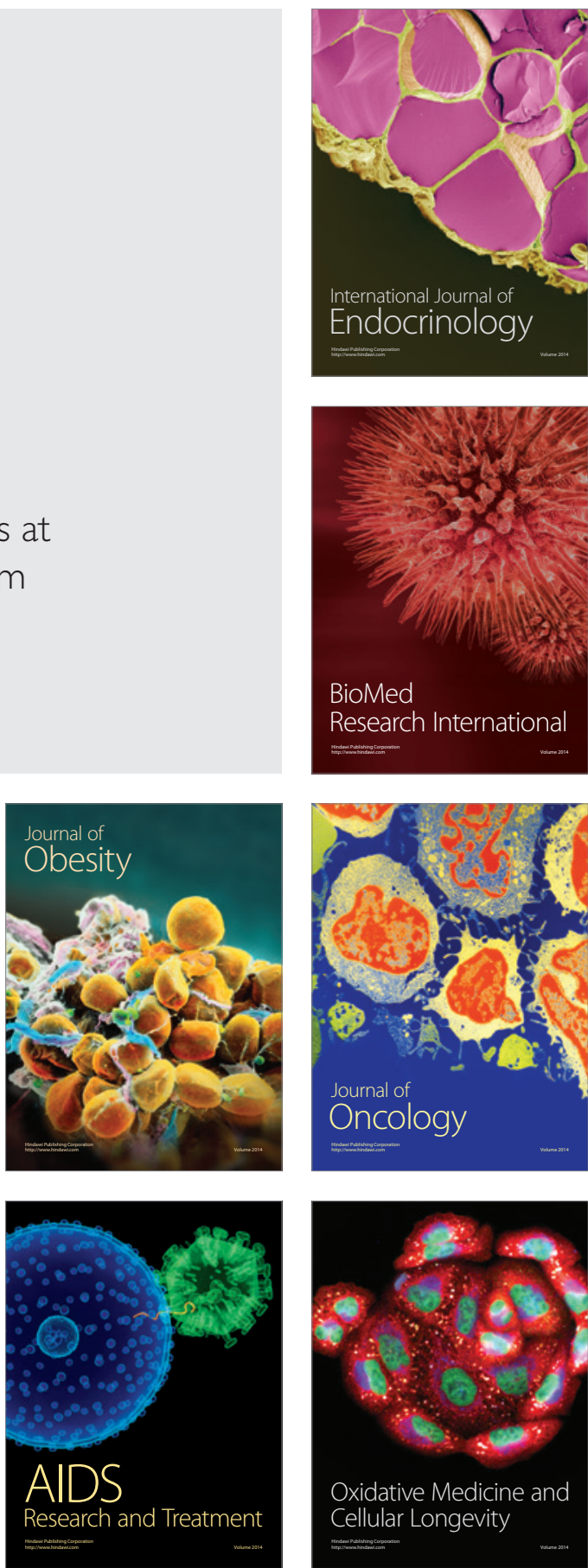